\title{
Mode discernibility and bounded-error state estimation for nonlinear hybrid systems *
}

\author{
Nacim Ramdani ${ }^{a}$ Louise Travé-Massuyès ${ }^{b}$ Carine Jauberthie ${ }^{b}$ \\ ${ }^{a}$ Univ. Orléans, INSA-CVL, PRISME, EA 4229, F45072, Orléans, France \\ ${ }^{\mathrm{b}}$ CNRS, LAAS, University of Toulouse, 31031 Toulouse, France
}

\begin{abstract}
State estimation is a key engineering problem when addressing control or diagnosis issues for complex dynamical systems. The issue is still challenging when the latter systems must be modelled as hybrid discrete-continuous dynamics, which is true for many complex and safety-critical systems. In this paper, we investigate nonlinear hybrid state estimation in a bounded-error framework using reliable and robust methods. We first establish a testable condition for current mode location discernibility. Then we build our hybrid state estimator which relies on a prediction-correction approach. An illustrative example is presented.
\end{abstract}

Key words: Bounded-error, hybrid systems, interval analysis, nonlinear systems, estimation, reachability, uncertain systems, zonotope.

\section{Introduction}

State estimation is a key engineering problem when addressing control or diagnosis issues with complex dynamical systems. Many systems exhibit both smooth continuous dynamics and abrupt switches, hence can be efficiently modeled using hybrid automata, which combine discrete and continuous variables (Alur et al., 1995). Hybrid state estimation aims at reconstructing both the discrete mode, hence the switching sequence, and the associated continuous state variables, based on a set of possibly discrete-time measurements, the knowledge of the hybrid model, and assumptions about the uncertainties and perturbations acting on the system. For instance, Wang et al. (2007) developed a robust exponentially ultimately bounded hybrid state observer using the unknown input extended Kalman observer for hybrid systems with discrete-time nonlinear dynamics, while Guo and Huang (2013) developed a moving horizon estimation scheme for switched systems and analyzed its stability under the uniform observability prop-

\footnotetext{
* This work is supported by the French National Research Agency under contract ANR 2011 INS 006 MAGICSPS (projects.laas.fr/ANR-MAGIC-SPS). This paper was not presented at any IFAC meeting. Corresponding author N. Ramdani.
}

Email address: nacim.ramdani@univ-orleans.fr (Nacim Ramdani). erty. Balluchi et al. (2013) addressed exponentially ultimately bounded observer design for hybrid systems with linear continuous-time dynamics, and Barhoumi et al. (2012) addressed the synthesis of high gain observers for uniformly observable nonlinear hybrid systems.

In this paper, we address hybrid state estimation in the unknown-but-bounded-error (UBBE) framework, where one assumes that all uncertain quantities, not only measurement noise but model uncertainty and modelling errors belong to a known bounded set with no other assumption about the distribution within the set (Schweppe, 1968; Milanese et al., 1996). In many cases, the UBBE assumption is natural and straightforward, and it requires less data than any statistical assumptions. In the UBBE framework, the estimation problem no longer has a unique solution, but there exists a set of state vectors that are consistent with measured data, the model structure and the prior error bounds. Then, set-membership estimation (SME) techniques allow the derivation of a conservative outer-approximation of the set of consistent state vectors at each time instant. There has been a significant research effort related to SME with continuous systems and the developed approaches may be sorted in two main types. One type of methods focus on the design of Luenberger-like interval observers, which assume the availability of continuous measurements (a.o. (Gouzé et al., 2000; Raïssi et al., 2012; Efimov et al., 2013; Mazenc and Dinh, 2014; Tha- 
bet et al., 2014; Mazenc et al., 2015)). Another type of methods use and extend the predictor-corrector estimation scheme as encountered in the Kalman filter (Xiong et al., 2013). For nonlinear systems, Kieffer et al. (2002) developed the first predictor-corrector based SME approach for discrete-time systems using interval analysis, then Jaulin (2002); Raïssi et al. (2004); Meslem et al. (2010); Meslem and Ramdani (2011) extended the approach to handle state estimation for continuous-time systems with discrete measurements by combining interval analysis and reachability computation capabilities as obtained using guaranteed solving tools for interval initial value problems (IVP) for nonlinear ordinary differential equations (ODE). This paper is in line with the second set of methods and aims at extending the predictor-corrector-based SME approach to truly nonlinear hybrid continuous-discrete dynamical systems with discrete measurements, thus developing an SME technique to simultaneously reconstruct, at each time instant, the set of consistant system's switching sequence and the corresponding set of consistent continuous state vectors.

SME for truly nonlinear hybrid systems is a challenging issue that has attracted only few researchers. To the best of our knowledge, the only works addressing this issue are by Benazera and Travé-Massuyès (2009), who addressed hybrid systems with discrete-time only nonlinear continuous dynamics, and Eggers et al. (2012) who investigated the feasibility of using satisfiability checkers. Clearly, if one knew in which mode the hybrid system is operating, the estimation of the continuous component of the hybrid system would merely make use of the existing SME algorithms for continuous systems. Therefore, the main ingredient of our SME for hybrid systems is the ability to distinguish the current active location mode from the observation of the input-output behaviour. To the best of our knowledge, the observability and detectability of hybrid systems have been studied only for linear switching systems (Babaali and Pappas, 2005; De Santis et al., 2003, 2009; Fliess et al., 2008; De Santis, 2011; De Santis and Di Benedetto, 2017; Lou and Yang, 2011). In this paper we introduce a new computable condition for analyzing mode discernibility for the general class of nonlinear hybrid systems. We say that two location modes are discernible if there exists a control making it possible to distinguish them by their outputs. In the case of autonomous systems, the output trajectories must differ at some point in time. Then, using an one-parameter-tuned composite continuous model, we show that the identifiability of the tuning parameter implies current mode discernibility. The contribution of this paper is twofold. First, we give a computable condition for current mode discernibility, then we build a predictor-corrector-type scheme for SME of the complete state of general class of hybrid systems, in the UBBE framework.

The paper is structured as follows: Sect. 2 defines hybrid dynamical systems, while Sect. 3 formulates the estima- tion problem. Sect. 4 introduces our approach for current mode discernibility analysis, while Sect. 5 describes the complete state set-membership estimation. Sect. 6 discusses method complexity and convergence. Sect. 7 reports the numerical evaluation on a realistic example, before conclusions.

\section{Hybrid dynamical systems}

Hybrid dynamical systems (HDS) can be represented by a hybrid automaton (Alur et al., 1995) given by

$$
H A=(\mathbb{Q}, \mathbb{Z}, \mathbb{U}, \mathbb{F}, \operatorname{Inv}, \Sigma, \Psi, \mathbb{G}, \mathbb{A}),
$$

where: $\mathbb{Q}=\{q\}$ is a set of locations, i.e. discrete state or modes; domain $\mathbb{Z} \subseteq \mathbb{R}^{n}$ is the definition domain of the continuous component with dimension $n$ that may depend on $q$; domain $\mathbb{U} \subseteq \mathbb{R}^{n_{u}}$ is the set of admissible control inputs; $\mathbb{F}=\left\{f_{q}\right\}$ is the set of non-autonomous differential equations characterizing flow transition in mode $q$, of the form

$$
\text { flow }(q): \quad \dot{z}(t)=f_{q}(z(t), u(t)),
$$

where $f_{q}: \mathbb{Z} \times \mathbb{U} \mapsto \mathbb{Z}$ is a nonlinear function assumed sufficiently smooth over $\mathbb{D} \subseteq \mathbb{R}^{n}$; Inv is an optional invariant, which assigns a domain to the continuous state space of each location:

$$
\operatorname{Inv}(q): \quad \nu_{q}(z(t))<0,
$$

where inequalities are taken componentwise, $\nu_{q}: \mathbb{Z} \mapsto$ $\mathbb{R}^{m}$ is also nonlinear, and the number $m$ of inequalities may also depend on $q ; \Sigma$ is a set of exogenous events; $\Psi=\left\{\rho_{e}\right\}_{e \in \mathbb{A}}$ is the set of reset maps, taken as continuous nonlinear functions; $\mathbb{G}=\left\{\gamma_{e}\right\}_{e \in \mathbb{A}}$ is the set of guard conditions of the form:

$$
\operatorname{guard}(e): \quad \gamma_{e}(z(t))=0
$$

where $\gamma_{e}():. \mathbb{Z} \mapsto \mathbb{R}^{m^{\prime}}$ is a nonlinear continuous function; $\mathbb{A} \subseteq \mathbb{Q} \times \mathbb{Q}$ is the set of discrete transitions $\{e=$ $\left.\left(q \rightarrow q^{\prime}\right)\right\}$ given by the 5 -uple $\left(q\right.$, guard, $\left.s q, \rho_{e}, q^{\prime}\right)$, where $q$ and $q^{\prime}$ represent upstream and downstream locations respectively, $s q \in \Sigma, \rho_{e} \in \Psi$, and guard $\in \mathbb{G}$. A transition $q \rightarrow q^{\prime}$ occurs when the continuous state flow reaches the guard set, i.e. when the continuous state satisfies condition (4).

Let us also consider the following measurement equation

$$
\operatorname{output}(q): \quad y(t)=\mu_{q}^{\top} z(t),
$$

where $\mu_{q} \in \mathbb{R}^{n \times n_{y}}$, depends on mode $q$.

Let us now recall the concept of hybrid trajectory (or hybrid solution). Les us consider a finite time horizon 
$\left[t_{0}, t_{N}\right]$ and denote $\chi\left(t_{0}\right)=\left(q_{0}, z_{q_{0}}\left(t_{0}\right)\right)$ the initial hybrid state. We can define as in continuous dynamics,

$$
z_{q_{0}}\left(t ; t_{0}, \chi\left(t_{0}\right)\right)
$$

the continuous state vector solution of the initial value problem (IVP) for the continuous ordinary differential equation (ODE) (2) starting from the initial state vector $z_{q_{0}}\left(t_{0}\right)$ at time $t_{0}$ in mode $q_{0}$. A discrete transition $e=q_{0} \rightarrow q_{1}$ occurs when the continuous flow trajectory intersects the guard set at time $t_{e}$, i.e. $\exists t_{e} \geq$ $t_{0}, \gamma_{e}\left(z_{q_{0}}\left(t_{e}\right)\right)=0$. Then, the continuous state vector is reset as $z_{q_{1}}\left(t_{e}^{+}\right)=\rho_{e}\left(z_{q_{1}}\left(t_{e}^{-}\right)\right)$. The switching sequence for $\operatorname{HDS}(2-5)$ may be written in the general case of $M$ discrete transitions as

$$
s e q=\left\{\left(t_{0}, q_{0}\right),\left(t_{e_{1}}, q_{1}\right),\left(t_{e_{2}}, q_{2}\right), \ldots,\left(t_{e_{M}}, q_{M}\right)\right\} .
$$

In fact, at each time instant $t \in\left[t_{0}, t_{N}\right]$, we can define the hybrid solution trajectory of the hybrid system (2$5)$ starting from the continuous state vector $z_{q_{0}}\left(t_{0}\right)$ at $t_{0}$ in the discrete mode $q_{0}$ as

$$
\chi\left(t ; t_{0}, \chi\left(t_{0}\right)\right)=\left(q_{i}(t), z_{q_{i}(t)}\left(t ; t_{e_{i}}, \chi\left(t_{e_{i}} ; t_{0}, \chi\left(t_{0}\right)\right)\right)\right),
$$

where $t_{e_{i}}$ is a switching time instant such that $t_{e_{i}} \leq t \leq$ $t_{e_{i+1}}, e_{i} \in \mathbb{A}$. We can also define the HDS output by

$$
\left.y_{q_{i}(t)}\left(t ; t_{0}, \chi\left(t_{0}\right)\right)=\mu_{q_{i}(t)}^{\top} z_{q_{i}(t)}\left(t ; t_{0}, \chi\left(t_{0}\right)\right)\right)
$$

where $z_{q_{i}(t)}(t)$ is the continuous component of $\chi(t)$. Now, let us consider the set $\chi_{0}=\mathbb{Q}_{0} \times \mathbb{Z}_{0}$ of possible initial hybrid state $\chi\left(t_{0}\right)$, cartesian product of the set of possible initial discrete modes $\mathbb{Q}_{0}$ and the the bounded initial domain $\mathbb{Z}_{0}$ of $z_{q_{0}}\left(t_{0}\right)$ when in any mode $q_{0} \in \mathbb{Q}_{0}$. We can extend (8)-(9) to set-valued initial conditions, as follows

$$
\begin{aligned}
& \chi\left(t ; t_{0}, \chi_{0}\right)=\left\{\chi\left(t ; t_{0}, \chi\left(t_{0}\right)\right) \mid \chi\left(t_{0}\right) \in \chi_{0}\right\} \\
& =\bigcup_{\chi_{0} \in \chi_{0}} \chi\left(t, t_{0}, \chi\left(t_{0}\right)\right) \\
& \begin{array}{r}
\mathcal{Y}\left(t ; t_{0}, \chi_{0}\right)=\left\{y_{q(t)}\left(t ; t_{0}, \chi\left(t_{0}\right)\right) \mid \chi\left(t_{0}\right) \in \chi_{0}\right\} \\
=\bigcup_{\chi_{0} \in \chi_{0}} y_{q(t)}\left(t, t_{0}, \chi\left(t_{0}\right)\right)
\end{array}
\end{aligned}
$$

\section{Set-membership hybrid state estimation}

Let us now assume that measurements $\tilde{y}_{j}$ of the output vector are available at sampling times $t_{j} \in$ $\left\{t_{1}, t_{2}, \ldots, t_{n}\right\} \subset\left[t_{0}, t_{N}\right]$. Note that the sampling interval does not need to be constant. Let us denote by $\mathbb{E}_{j}=\left[-\varepsilon_{j}, \varepsilon_{j}\right]$ a feasible domain for the output error at time $t_{j}$, the feasible domain for model output at time $t_{j}$ is then given by :

$$
\mathbb{Y}_{j}=\tilde{y}_{j}+\mathbb{E}_{j}=\left[\tilde{y}_{j}-\varepsilon_{j}, \tilde{y}_{j}+\varepsilon_{j}\right] .
$$

Remark 1 In this paper, it is assumed that there is no outlier, i.e. the true system output lays inside the domains (12).

The goal of bounded error estimation is to compute conservative outer enclosures for feasible sets for both the discrete modes and associated continuous variables that are consistent with the feasible domains for measurements and the uncertain hybrid model. That is, given two measurements $\mathbb{Y}_{j}$ and $\mathbb{Y}_{j+1}$ gathered at the two time instants $t_{j}$ and $t_{j+1}$, the estimation problem boils down to simultaneously, for all $j, 0 \leq j \leq N-1$ :

(i) Reconstruct all feasible switching sequences seq. This requires to detect and identify all possible discrete transitions $e \in \mathbb{A}$ that may occur at time $t_{e} \in\left[t_{j}, t_{j+1}\right]$. Because of the uncertainties, there might be a continuum of time instants where events may occur. There may also exist several such time intervals in $t_{e} \in\left[t_{j}, t_{j+1}\right]$.

(ii) Reconstruct the set

$$
\chi_{j}=\mathbb{Q}_{j} \times \bigcup_{q \in \mathbb{Q}_{j}} \mathbb{Z}_{q, j}
$$

of hybrid states solutions $\chi\left(t_{j}\right)=\left(q\left(t_{j}\right), z\left(t_{j}\right)\right)$ at time $t_{j}$, and the set

$$
\chi_{j+1}=\mathbb{Q}_{j+1} \times \bigcup_{q \in \mathbb{Q}_{j+1}} \mathbb{Z}_{q, j+1}
$$

of hybrid state solutions $\chi\left(t_{j+1}\right)=\left(q\left(t_{j+1}\right), z\left(t_{j+1}\right)\right)$ at time $t_{j+1}$ that are consistent with the switching sequence reconstructed in (i), the $\operatorname{HDS}(2-5)$, and the assumptions $\forall q\left(t_{j}\right) \in \mathbb{Q}_{j}, y_{q\left(t_{j}\right)}\left(t_{j}\right) \in \mathbb{Y}_{j}$ and $\forall q\left(t_{j+1}\right) \in \mathbb{Q}_{j+1}, y_{q\left(t_{j+1}\right)}\left(t_{j+1}\right) \in \mathbb{Y}_{j+1}$, where $\mathbb{Q}_{k}$ is the set of possible modes and $\mathbb{Z}_{q, k}$ is the set of continuous state vectors at time $t_{k}$.

\section{Mode discernibility}

Here, we state our first result on current mode discernibility. We show that the latter is addressed via parametric identifiability. We hence first introduce key concepts about identifiability.

\subsection{Parameter identifiability}

Let us consider a controlled dynamical system described by the ODE

$$
\dot{z}=\mathfrak{f}(z, p, u),
$$


and the output equation

$$
y=\mathfrak{g}(z, p),
$$

where $z(t) \in \mathbb{R}^{n}, p \in \mathbb{P} \subseteq \mathbb{R}^{n_{p}}$ is a parameter vector and $u(t) \in \mathbb{R}^{n_{u}}$ a control input. Here we assume the mappings $\mathfrak{f}$ and $\mathfrak{g}$ are real, analytic and infinitely differentiable on $\mathbb{M}$, where $\mathbb{M}$ is an open set of $\mathbb{R}^{n}$. Let us consider $t \in\left[t_{0}, T\right]$ where $T$ is a finite or infinite time bound. Parameter identifiability is defined as follows by Ljung and Glad (1994).

Definition 4.1 The parameter $p_{i}$ of model (15)-(16) is globally identifiable if there exists $u(t) \in \mathbb{R}^{n_{u}}$ such that for all $\left(\hat{p}_{i}, p_{i}^{*}\right) \in \mathbb{P}^{2}, \hat{p}_{i} \neq p_{i}^{*}$ :

$$
\left(\forall t \in[0, T], y\left(t, \hat{p}_{i}, u\right)=y\left(t, p_{i}^{*}, u\right)\right) \Rightarrow\left(\hat{p}_{i}=p_{i}^{*}\right),
$$

and the parameter vector $p$ is globally identifiable in $\mathbb{P}$ if all its components $p_{i}$ are globally identifiable in $\mathbb{P}^{n_{p}}$.

Identifiability of the parameter vector $p$ can be tested via differential algebra. The method consists in eliminating state variables to obtain relations linking outputs, inputs (if any), and parameters. For doing this, one can use the Rosenfeld-Groebner algorithm implemented in the package DifferentialAlgebra of Maple (Boulier et al., 1997) with the elimination order $\{p\}<\{y, u\}<\{x\}$ (Kolchin, 1973). Among the solutions delivered by the algorithm, one is called the characteristic presentation because it corresponds to the general solution, the other ones being particular solutions. The characteristic presentation contains differential polynomials linking outputs, inputs (if any), and parameters of the form:

$$
\begin{aligned}
& R_{i}(y, u, p)=m_{0}^{i}(y, u)+\sum_{k=1}^{n_{i}} \theta_{k}^{i}(p) m_{k}^{i}(y, u) \\
& i=1, \ldots, n_{y}
\end{aligned}
$$

where $\left(\theta_{k}^{i}(p)\right)_{1 \leq k \leq n_{i}}$ are rational in $p, \theta_{u}^{i} \neq \theta_{v}^{i}(u \neq$ $\left.v), m_{k}^{i}(y, u)\right)_{0 \leq k \leq n_{i}}$ are differential polynomials with respect to $y, u$ and $m_{0}^{i}(y, u) \neq 0$.

Definition $4.2\left\{\theta_{k}^{i}(p)\right\}_{1 \leq k \leq n_{i}}$ is called the exhaustive summary of $R_{i}$.

The size of the system is the number of outputs. For the time being, we assume that $n_{y}=1$, that is, there is one output and we rewrite $n_{1}=\bar{n}, R_{1}=R, m_{k}^{1}(y, u)=$ $m_{k}(y, u)$.

Let us consider $\Delta R(y, u)$ that denotes the functional determinant formed from the $\left\{m_{k}(y, u)\right\}_{1 \leq k \leq \bar{n}}$ and given by the Wronskian

$$
\Delta R(y, u)=\left|\left(\begin{array}{ccc}
m_{1}(y, u) & \ldots & m_{n}(y, u) \\
m_{1}(y, u)^{(1)} & \ldots & m_{n}(y, u)^{(1)} \\
& \ddots & \\
& \ldots & m_{n}(y, u)^{(\bar{n}-1)}
\end{array}\right)\right| .
$$

Theorem 1 (Denis-Vidal et al. (2001)) Assume that the functional determinant $\Delta R(y, u)$ is not identically equal to zero ${ }^{1}$. If the mapping

$$
\varphi: p \mapsto\left(\theta_{1}(p), \ldots, \theta_{n}(p)\right)
$$

is injective then the parameter $p$ is globally identifiable.

When there are more than one outputs, i.e. $n_{y} \geq 1$, for each of the $n_{y}$ obtained differential polynomials $R_{i}(y, u, p), i=1, \ldots, n_{y}$, the functional determinant is evaluated. If it is not identically equal to zero, the associated exhaustive summary is added to the image of the function $\varphi$ for which injectivity has to be studied.

\subsection{Mode discernibility}

We can now establish our contribution regarding mode discernibility. We first foster on the following definition of mode discernibility adapted from Babaali and Pappas (2005) and rewritten using notation of Sect. 2.

Definition 4.3 (Mode discernibility) Two different modes $q_{1}$ and $q_{2}$ are discernible over $T>0$ if whenever $q([0, T]) \equiv q_{1}$ and $q^{\prime}([0, T]) \equiv q_{2}$,

$$
\begin{aligned}
& q_{1} \neq q_{2} \Rightarrow \exists u, \forall \chi_{0}, \forall \chi_{0}^{\prime}, \\
& \quad y_{q}\left([0, T] ; 0, \chi_{0}, u\right) \neq y_{q^{\prime}}\left([0, T] ; 0, \chi_{0}^{\prime}, u\right) .
\end{aligned}
$$

where the output vectors $y_{q}$ and $y_{q^{\prime}}$ in (19) are written using the notation of (9).

In words, location modes $q_{1}$ and $q_{2}$ are discernible if there exists a control making it possible to distinguish them by their outputs.

Let us introduce the integer variable $s \in \mathbb{Q}$ and define the composite continuous model,

$$
\dot{z}(t)=\mathcal{F}(z(t), s, u(t)),
$$

\footnotetext{
1 This assumption consists in verifying the linear independence of the $m_{k}(y, u), k=1, \ldots, n$. For doing this, it is sufficient to find a time point at which the Wronskian is non-zero. In the framework of differential algebra, this condition consists in verifying that this functional determinant is not in the ideal obtained after eliminating state variables. In practice, it can be checked with the function Belong_To of the package DifferentialAlgebra of Maple 16.
} 
where $z \in \mathbb{Z}$. The mapping $\mathcal{F}: \mathbb{Z} \times \mathbb{Q} \times \mathbb{U} \mapsto \mathbb{Z}$ is built using the collection $\mathbb{F}$ of the hybrid plant $H A$ (1) subsystems, and the set of locations $\mathbb{Q}$ as follows

$$
\mathcal{F}(z, s, u)=\sum_{i=1}^{n_{q}} \frac{\Pi_{j=1, j \neq i}^{n_{q}}\left(s-q_{j}\right)}{\Pi_{j=1, j \neq i}^{n_{q}}\left(q_{i}-q_{j}\right)} f_{q_{i}}(z, u)
$$

where $n_{q}$ is the cardinality of set $\mathbb{Q}$. Let us define $z_{s}\left(t, t_{0}, z_{0}, u\right)$ the solution of IVP for ODE (20) starting from initial conditions $z_{0}$ at $t_{0}$, We can now define a composite output model

$$
\mathcal{Y}_{s}\left(t, t_{0}, z_{0}, u\right)=\sum_{i=1}^{n_{q}} \frac{\Pi_{j=1, j \neq i}^{n_{q}}\left(s-q_{j}\right)}{\Pi_{j=1, j \neq i}^{n_{q}}\left(q_{i}-q_{j}\right)} \eta_{q_{i}}^{\top} z_{s}\left(t, t_{0}, z_{0}, u\right)
$$

Remark 1 It is straightforward to notice that for any $i \in\left\{1, \ldots, n_{q}\right\}$, whenever $s=q_{i}$, the composite system (20)-(21) operates in mode $q_{i}$ with the appropriate output model (22).

Theorem 2 If the scalar parameter $s$ in system (20)(22) is identifiable, then modes $q_{i}, i \in\left\{1, \ldots, n_{q}\right\}$, of $H D S$ (1) are discernible.

Proof 1 If parameter $s$ is identifiable, then it exists $u$ such that for any $s_{1} \equiv q_{1}$ and $s_{2} \equiv q_{2}$ in $\mathbb{Q}$, one has $s_{1} \neq s_{2} \Rightarrow\left(\forall z_{0}, \forall z_{0}^{\prime}, \exists t \in[0, T], \mathcal{Y}_{s_{1}}\left(t, t_{0}, z_{0}, u\right) \neq\right.$ $\left.\mathcal{Y}_{s_{2}}\left(t, t_{0}, z_{0}^{\prime}, u\right)\right), \Leftrightarrow\left(\forall z_{0}, \forall z_{0}^{\prime}, \quad \mathcal{Y}_{s_{1}}\left([0, T], t_{0}, z_{0}, u\right) \neq\right.$ $\left.\mathcal{Y}_{s_{2}}\left([0, T], t_{0}, z_{0}^{\prime}, u\right)\right)$. The modes $q_{1}$ and $q_{2}$ are discernible. This holds for any pair, hence all the modes $q_{i} \in \mathbb{Q}$ are discernible.

Remark 2 Note that in our case the identifiability condition of Theorem 1 needs to be checked for the parameter $p$ being scalar and given by the only parameter $s$.

Remark 3 Theorem 2 does not consider mode invariants that may be used to discriminate two different modes. It is thus not a necessary condition for mode discernibility.

\section{A Predictor-Corrector approach to complete state estimation}

In the sequel, we solve the estimation problem by using the prediction-correction approach we extend to hybrid dynamical systems.

For the prediction step, we rely on the hybrid reachability method of (Maïga et al., 2016), and denoted Hybrid_Reach(.) in the sequel. This method combines interval Taylor methods and zonotope enclosures to bound the solution set of the IVP ODE at time $t_{j}$ for each active location model. In the UBBE framework, there can be several location modes reconstructed by the estimator, therefore the forward reachable set may be characterized by a union of these bounding zonotopes. Given a set of consistent hybrid states $\chi_{j}$ reconstructed at time $t_{j}$, the prediction step computes the forward reachable set at time $t_{j+1}$,

$$
\chi_{j+1}^{F}=\text { Hybrid_Reach }\left(t_{j+1} ; t_{j}, \chi_{j}\right) \text {. }
$$

In the correction stage, we use set computations with zonotopes to filter the forward image at time $t_{j+1}$. Using measurement domains $\mathbb{Y}_{j+1}$ that are available at time $t_{j+1}$, we reduce the domain of $\chi_{j+1}^{F}$ by removing parts inconsistent with the model, the actual data and the error bounds. More formally, for any reachable discrete mode $q \in \mathbb{Q}_{j+1}^{F}$, where $\mathbb{Q}_{j+1}^{F}$ is the set of forward reachable discrete modes, we filter the associated forward continuous reachable set $\mathbb{Z}_{q, j+1}^{F}$ to obtain the set of consistent continuous states in mode $q$. Considering (12) and (5) for each mode, the set of continuous state vectors consistent with the feasible domain for model output at time $t_{j+1}$ is the strip

$$
\mathcal{S}_{q, j+1}=\left\{z_{q} \in \mathbb{Z}|| \eta_{q}^{\top} z_{q}-\tilde{y}_{j+1} \mid \leq \varepsilon_{j+1}\right\} .
$$

Therefore, for each mode $q$, the state vectors consistent with the model, the actual data and the error bounds are given by

$$
\mathbb{Z}_{q, j+1}^{\cap}=\mathcal{S}_{q, j+1} \cap \mathbb{Z}_{q, j+1}^{F},
$$

We prune off the inconsistent discrete modes, those for which intersection computed in (25) is empty, and keep only the consistent discrete modes. The latter are gathered in set

$$
\mathbb{Q}_{j+1}^{\cap}=\left\{q \in \mathbb{Q}_{q, j+1}^{F} \mid \mathbb{Z}_{q, j+1}^{\cap} \neq \emptyset\right\} .
$$

Finally the consistent hybrid states $\chi_{j+1}$ are simply computed as the union of hybrid states with consistent discrete modes,

$$
\chi_{j+1}=\bigcup_{q \in \mathbb{Q}_{j+1}^{n}}\left(q, \mathbb{Z}_{q, j+1}^{\cap}\right) .
$$

The algorithm discards inconsistent discrete modes and prunes inconsistent continuous state vectors in each consistent discrete mode, as illustrated on Fig. 1.

The correction step first checks for each forward reachable mode if intersection (25) is not empty. This is done by algorithm Test_ZIS(.) which implements a testable condition established by Vicino and Zappa (1996). If the intersection is not empty, the zonotope of minimal size that bounds the intersection (25) is computed by algorithm Zono_Inter(.). The latter implements the explicit solution etablished by Alamo et al. (2005). The proposed approach is summarized in Algorithm 1 "Predictor Corrector Hybrid Set-Membership Estimation". 


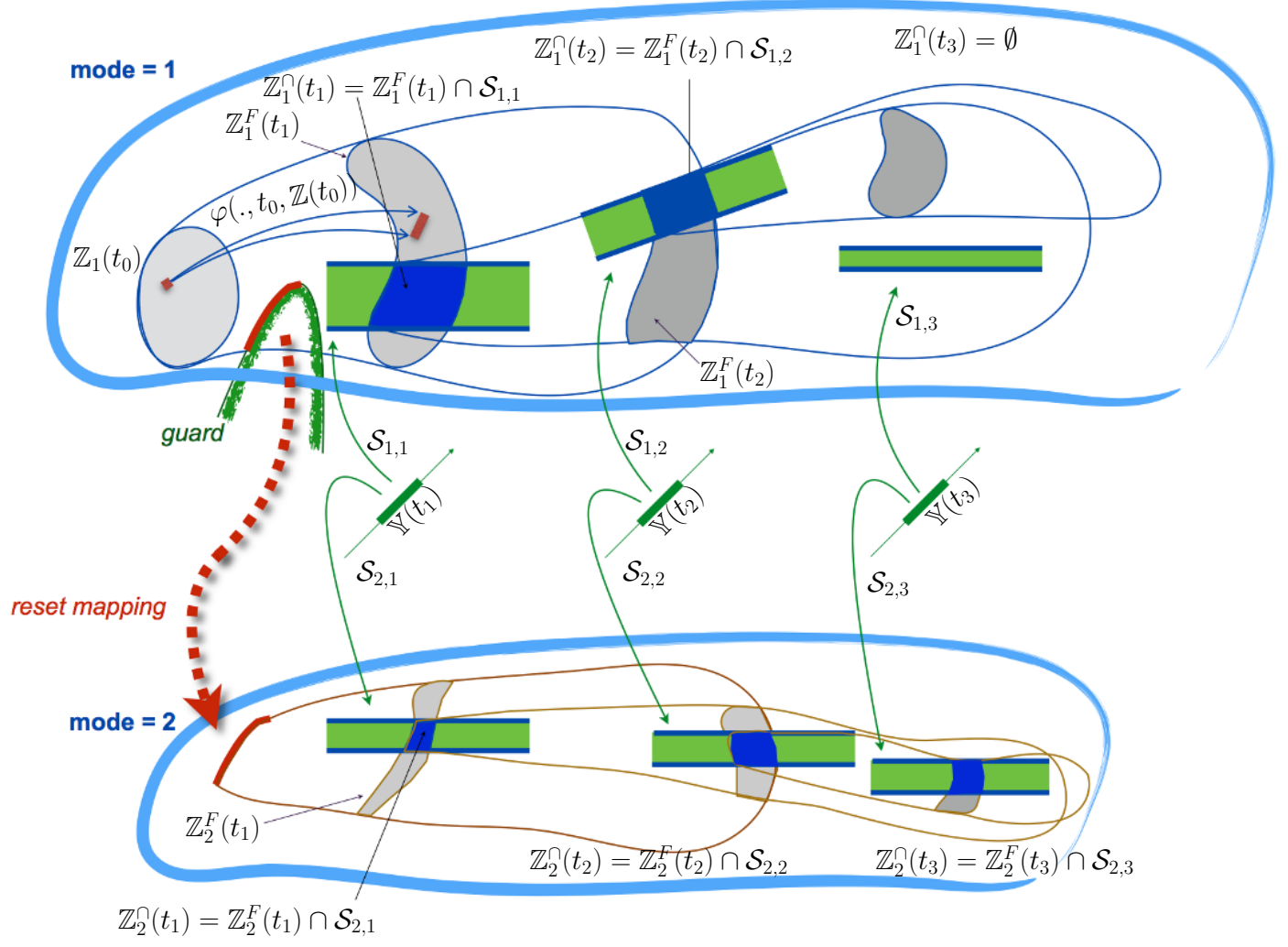

Fig. 1. Predictor-corrector approach to set-membership hybrid estimation. Using hybrid reachability computation, the hybrid state $\left(q=1, \mathbb{Z}_{1}^{F}\left(t_{1}\right)\right) \cup\left(q=2, \mathbb{Z}_{2}^{F}\left(t_{1}\right)\right)$ reachable at time $t_{1}$ starting from domain $\left(q=1, \mathbb{Z}_{1}\left(t_{0}\right)\right)$ at time $t_{0}$, is computed while correctly detecting the discrete transition mode 1 to mode 2 which occurs during time interval $\left[t_{0}, t_{1}\right]$. In each mode, the inconsistent continuous state vectors are pruned off by computing the intersection with the strip $\mathcal{S}_{q, 1}$ characterizing the continuous state vectors consistent with datum domain $\mathbb{Y}\left(t_{1}\right)$. Resuming from the new domains in each mode, i.e. $\left(q=1, \mathbb{Z}_{1}\left(t_{1}\right)\right) \cup\left(q=2, \mathbb{Z}_{2}\left(t_{1}\right)\right)$ the approach eventually discards mode 1 at time $t_{3}$ since the continuous set reachable at time $t_{3}$ does not intersect strip $\mathcal{S}_{1,3}$ characterizing datum $\mathbb{Y}\left(t_{3}\right)$ in mode 1 . The approach resumes from $\left(q=2, \mathbb{Z}_{2}\left(t_{3}\right)\right)$ with the single mode 2 , which is the discrete mode consistent with the model, the error bounds and actual data.

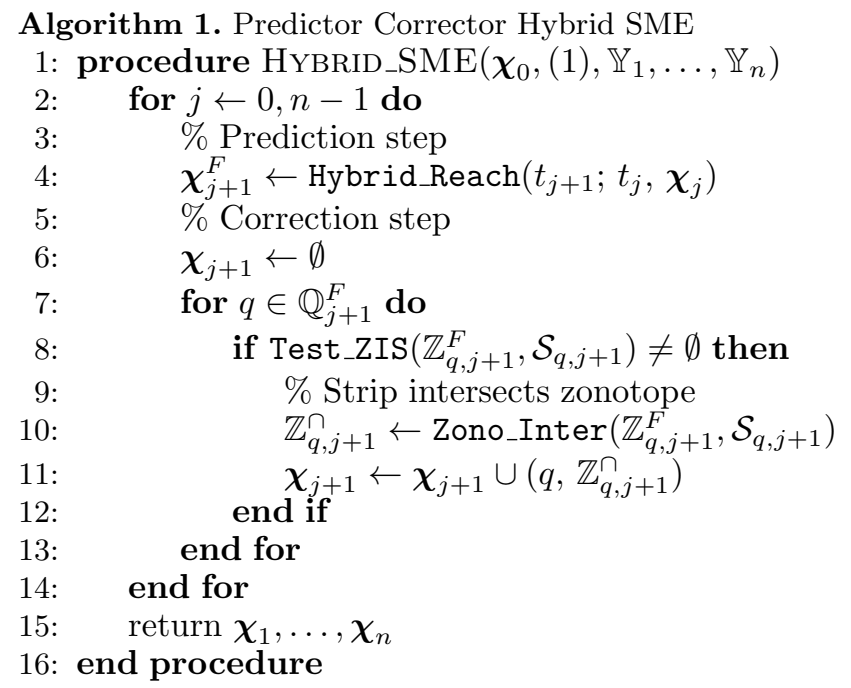

\section{Convergence and method complexity}

The convergence of predictor-corrector set-membership estimation methods is related to the properties of guar- anteedness and arbitrary precision. The guaranteedness property is the fact that the estimated set for the hybrid state vectors is guaranteed to enclose the actual state vector of the system. The property of arbitrary precision means that the estimated set converges from the outside to the actual state vector. These issues have been well-discussed for nonlinear discrete-time continuous systems, see (Kieffer et al., 2002) for details. For hybrid systems as studied in the present paper, the convergence property of the correction step is ensured since the correction step uses analytical expressions and the size of the bounding zonotopes is minimized at each time step in each discrete mode. Regarding the convergence property of the prediction step, i.e. the hybrid reachability computation, the proposition below from (Maïga et al., 2016) addresses guaranteedness convergence property of hybrid reachability computation. It stems from the use of guaranteed numerical tools.

Proposition 1 (Conservative hybrid reachability) The hybrid reachability algorithm implemented in the 
Hybrid-Reach function (23) from (Mä̈ga et al., 2016) provides guaranteed outputs, i.e. the flow-pipe generated by alternating continuous reachability, flow guard intersection, and reset mappings is guaranteed in the sense that it encloses all the trajectories and detects all events of the HDS (2)-(5) that are consistent with the uncertainty domains of the initial hybrid state $\mathbb{Q}_{0} \times \mathbb{Z}_{0}$.

To the best of our knowledge, there are no theoretical results available regarding the arbitrary precision property for nonlinear hybrid reachability computation. The overall complexity of the predictor-corrector approach stems mainly from the complexity of continuous reachability and event detection and localisation in hybrid reachability, and from the analytical expressions used in the correction step. Continuous reachability uses an interval Taylor series method, which is of polynomial complexity. Furthermore, although solving constraint satisfaction problems (CSP) in event detection and localisation is in theory NP-hard, there have been efforts to develop solving techniques whose practical time complexity is better than the exponential worst case (Tuy, 1995).

\section{Numerical evaluation}

For the experimentation purpose of this paper, we consider a hybrid damped double mass-spring system where one of the dampers is only active when the absolute value of the velocity magnitude of one mass is greater than a given threshold $v_{0}$. Otherwise, the given mass motion is not damped. This system may represent a vehicle suspension system (Koch and Kloiber, 2014). The hybrid dynamical system obtained is modelled by $\dot{x}(t)=$ $\left.f_{q}(x(t), p), x(t)\right) \in \mathbb{R}^{4}$, three modes $q \in \mathbb{Q}=\{0,1,2\}$ and four transitions, i.e. using notations of (1) with $u=0$ and $z=(x, p), f_{q}(x, p)=A_{q}(p) x, q \in\{0,1,2\}$, where matrix $A_{q}(p)$ is given by

$$
A_{q}(p)=\left[\begin{array}{cccc}
0 & 1 & 0 & -1 \\
-p_{1} & -p_{2} \zeta(q) & 0 & p_{2} \zeta(q) \\
0 & 0 & 0 & 1 \\
p_{3} & p_{4} \zeta(q) & -p_{5} & -\left(p_{4} \zeta(q)+p_{6}\right)
\end{array}\right]
$$

with $q \mapsto \zeta(q)$ defined as: $\zeta(0)=0$, and $\zeta(q)=1$ if $q>$ 0 . Invariant, guard and reset functions are given by

$$
\begin{aligned}
& \left\{\begin{array}{l}
\nu_{0}(x, p)=\left(x_{2}-v_{0}\right) \wedge\left(-\left(x_{2}+v_{0}\right)\right), \\
\nu_{1}(x, p)=-\left(x_{2}-v_{0}\right), \\
\nu_{2}(x, p)=x_{2}+v_{0},
\end{array}\right. \\
& \gamma_{e}(x, p)=\left\{\begin{array}{l}
x_{2}+v_{0}, e=0 \rightarrow 2,2 \rightarrow 0 \\
x_{2}-v_{0}, e=0 \rightarrow 1,1 \rightarrow 0
\end{array}\right.
\end{aligned}
$$

where the threshold $v_{0}=0.1$ is known, and $\rho_{e}(x, p)=$ $x, \forall e$. We consider an uncertain parameter vector with very large relative uncertainties, ranging from $10 \%$ to $80 \%: p \in \mathbb{P}=[0.9,1.1] \times[0.035,0.235] \times[0.82,1.02] \times$ $[0.02,0.22] \times[0.67,0.87] \times[0.6,0.8]$. The output equation (5) $y(t)=\mu_{q}^{\top} x(t)$ is implemented with

$$
\mu_{q}^{\top}=\left[\begin{array}{llll}
1 & 0 & 0 & 0 \\
0 & 0 & 1 & 0
\end{array}\right], \quad q \in\{0,1,2\}
$$

\subsection{Mode discernibility analysis}

Before proceeding with hybrid estimation, it is important to know which modes are discernible from a structural point of view. This analysis is performed using the results of Sect. 4. In our case study, it is easy to see that modes $q_{1}$ and $q_{2}$ are not discernible; they have indeed the same dynamics and the same output equation. However, they can be distinguished using their invariant, since the two mode's invariants have empty intersection. The mode discernibility analysis is hence focused on discernibility of modes $q_{0}$ and $q_{1} / q_{2}$. The composite model $\Sigma_{q_{0} / q_{1}}$ of the form (20)-(22) for modes $q_{0}$ and $q_{1}$ is the following:

$$
\begin{aligned}
& \left\{\begin{aligned}
\dot{x}_{1}(t)= & x_{2}(t)-x_{4}(t), \\
\dot{x}_{2}(t)= & \frac{s-q_{1}}{q_{0}-q_{1}}\left(-p_{1} x_{1}(t)\right) \\
& +\frac{s-q_{0}}{q_{1}-q_{0}}\left(-p_{1} x_{1}(t)-p_{2} x_{2}(t)+p_{2} x_{4}(t)\right), \\
\dot{x}_{3}(t)= & x_{4}(t), \\
\dot{x}_{4}(t)= & \frac{s-q_{1}}{q_{0}-q_{1}}\left(p_{3} x_{1}(t)-p_{5} x_{3}(t)-p_{6} x_{4}(t)\right) \\
& +\frac{s-q_{0}}{q_{1}-q_{0}}\left(p_{3} x_{1}(t)+p_{4} x_{2}(t)-p_{5} x_{3}(t)\right.
\end{aligned}\right. \\
& \left\{\begin{aligned}
y_{1}(t)= & x_{1}(t) \\
y_{2}(t)= & x_{3}(t)
\end{aligned}\right.
\end{aligned}
$$

Running the Rosenfeld-Groebner algorithm, we get the characteristic presentation for the composite system $\Sigma_{q_{0} / q_{1}}$. Let us note that for the analysis, the parameter to be checked for identifiability is $s$. The modes $q_{0}$ and $q_{1}$ as well as the parameters $p_{1}$ to $p_{6}$ are considered as constants. The characteristic presentation contains the two following differential polynomials of the form (17) that link the outputs and the parameter $s$ :

$$
\left\{\begin{aligned}
R_{1}^{\Sigma_{q_{0} / q_{1}}}(y, s)= & \left(q_{0}-q_{1}\right) \ddot{y}_{1}(t)+q_{0}\left(p_{2}+p_{4}\right) \dot{y}_{1}(t) \\
& -s\left(p_{4}-p_{2}\right) \dot{y}_{1}(t) \\
& +\left(q_{0}-q_{1}\right)\left(p_{1}+p_{3}\right) y_{1}(t) \\
& +\left(q_{1}-q_{0}\right) p_{6} \dot{y}_{2}(t)+\left(q_{1}-q_{0}\right) p_{5} y_{2}(t), \\
R_{2}^{\Sigma_{q_{0} / q_{1}}}(y, s)= & \left(q_{0}-q_{1}\right) \ddot{y}_{2}(t)+\left(q_{0}-q_{1}\right) p_{6} \dot{y}_{2}(t) \\
& +\left(q_{0}-q_{1}\right) p_{5} y_{2}(t)+p_{4}\left(s-q_{0}\right) \dot{y}_{1}(t) p_{6} \\
& +\left(q_{1}-q_{0}\right) p_{3} y_{1}(t) .
\end{aligned}\right.
$$


Every differential polynomial has only one parameter bloc $\theta_{1}^{1}(s)=s$ and $\theta_{1}^{2}(s)=s$. For both, the functional determinant is not identically equal to zero provided that $\dot{y}_{1}(t) \neq 0$ and the mapping $\varphi$ of theorem 1 is injective. The parameter $s$ is hence globally identifiable. By theorem 2 , the modes $q_{0}$ and $q_{1}$ are therefore discernable (as well as the modes $q_{0}$ and $q_{2}$ ). Actually, one can easily solve formally $R_{1}(y, s)$ and $R_{2}(y, s)$ for the parameter $s$ and show that they provide the same result. If we assume that the system is in mode $q_{0}$ (resp. $q_{1}$ ), then the result should be $s=q_{0}$ (resp. $s=q_{1}$ ). Let us show this for mode $q_{0}$. For this purpose, let us consider the model $\Sigma_{q_{0}}$ for mode $q_{0}$ :

$$
\begin{aligned}
& \left\{\begin{array}{l}
\dot{x}_{1}(t)=x_{2}(t)-x_{4}(t), \\
\dot{x}_{2}(t)=-p_{1} x_{1}(t), \\
\dot{x}_{3}(t)=x_{4}(t), \\
\dot{x}_{4}(t)=p_{3} x_{1}(t)-p_{5} x_{3}(t)-p_{6} x_{4}(t) .
\end{array}\right. \\
& \left\{\begin{array}{l}
y_{1}(t)=x_{1}(t), \\
y_{2}(t)=x_{3}(t) .
\end{array}\right.
\end{aligned}
$$

and generate the corresponding input/output relations:

$$
\left\{\begin{array}{l}
R_{1}^{\Sigma_{q_{0}}}(y, s)=\ddot{y}_{1}(t)+\left(p_{1}+p_{3}\right) y_{1}(t)-p_{5} y_{2}(t)-p_{6} \dot{y}_{2}(t) \\
R_{2}^{\Sigma_{q_{0}}}(y, s)=\ddot{y}_{2}(t)-p_{3} y_{1}(t)+p_{5} y_{2}(t)+p_{6} \dot{y}_{2}(t) .
\end{array}\right.
$$

Substituting the expressions $\ddot{y}_{1}(t)$ and $\ddot{y}_{2}(t)$ obtained from $R_{1}^{\Sigma_{q_{0}}}(y, s)$ and $R_{2}^{\Sigma_{q_{0}}}(y, s)$ respectively, in one or the other of the relations $R_{1}^{\Sigma_{q_{0} / q_{1}}}(y, s)$ or $R_{2}^{\Sigma_{q_{0} / q_{1}}}(y, s)$ provides a unique solution for $s$ that is $s=q_{0}$. If the same is done for mode $q_{1}$, the obtained solution is also unique and given by $s=q_{1}$. This confirms that the injectivity condition of theorem 1 is satisfied. It hence confirms that the composite system $\Sigma_{q_{0} / q_{1}}$ is identifiable for $s$ and, by theorem 2 , that the modes $q_{0}$ and $q_{1}$ are discernable (as well as $q_{0}$ and $q_{2}$ ).

\subsection{Hybrid estimation}

We will now proceed with the hybrid estimation. The measurement time step is $t_{j+1}-t_{j}=h=0.15 \mathrm{~s}$, and time horizon is $\left[0, T_{\text {end }}\right]$, where $T_{\text {end }}=12 \mathrm{~s}$. The feasible domain for output error is taken as $\mathbb{E}_{j}=[-\varepsilon, \varepsilon]$, where $\varepsilon=0.025$ in scenario 1 , and $\varepsilon=0.05$ in scenario 2 . In both scenarios, the initial domain for the state vector is considered unknown, hence all modes in $\mathbb{Q}$ are taken active at $t_{0}$. Artificial data are generated with point parameter vector $p=\operatorname{mid}(\mathbb{P})$ and known initial state vector $x\left(t_{0}\right)=(1,0,0,0)$. The integration time step is chosen constant and the same as the measurement time step, also when running the state estimation Algorithm 1.

Fig. 2-(b-d,f-h) gather the reconstructed complete state vector for scenario $1(\varepsilon=0.025)$. The overall CPU time ${ }^{2}$ for the whole time duration is 242s. Fig. 2-(a,e) gather the reconstructed mode and $x_{2}$ state vector for scenario 2 $(\varepsilon=0.05)$. The overall CPU time is $3242 \mathrm{~s}$. In scenario 1 , the predictor-corrector algorithm Hybrid_SME( . ) starts with the three modes $\{0,1,2\}$, then prunes inconsistant modes after 2 time steps. In each operation mode, Hybrid_SME(.) reconstructs the feasible domains for the unmeasured components of the continuous state vector. Because the latter domains have non zero widths, the set of discrete modes reconstructed by Hybrid_SME(.) at a given time instant may include several modes, among which only one is the true mode, the others being spurious (because of structural non identifiability or because of uncertainty). This also exemplifies the conservativeness of our approach, i.e. the true solution is always captured. This behaviour is observed each time the system switches mode $\left(t_{j}=2.7, t_{j}=6, t_{j}=8.65\right)$. Finally, towards the end of the scenario, the magnitude of velocity $x_{2}$ varies slowly around zero, then since the reconstructed trajectory tube for $x_{2}$ has non-zero width, the tube covers the guard set during a large time interval. As a consequence, the discrete mode transition $e=0 \rightarrow 1$ remains activated and the reconstructed mode set is $\mathbb{Q}_{j}=\{0,1\}$. Nonetheless, the reconstructed tubes show widths that are consistent with the measurement errors. In scenario 2, the measurement noise magnitude is doubled. The reconstructed tubes show larger diameters than in scenario 1 , where diameters increase by factor 1.5 for $x_{1}, 1.7$ for $x_{2}, 2.1$ for $x_{3}$, and 1.8 for $x_{4}$. Hence, one observes spurious discrete mode reconstruction that lasts longer for every discrete transition. The true transitions are nonetheless always captured. Towards the end of the scenario, larger reconstructed tube for $x_{2}$ covers the guard set earlier than in scenario 1 . In its current implementation, algorithm Hybrid_Reach(.) merges, at each time instant, any union of trajectory tubes when the reconstructed mode is unique. When the reconstructed trajectory tubes cover the guard set, there are more than one reconstructed operation mode, hence tube merging is switched off. As a consequence, algorithm Hybrid_Reach(.) must compute with union of tubes more often, therefore requires larger CPU time in scenario $2(3242 \mathrm{~s})$ than in scenario $1(242 \mathrm{~s})$.

\section{Conclusion}

In this paper, we have introduced a new approach for analyzing current location mode discernibility with truly

\footnotetext{
${ }^{2}$ We use the Profil/Bias $\mathrm{C}++$ class for interval computation, the FABDAB++ package (www.fadbad.com) for automatic differentiation, AML++ (amlpp.sourceforge.net) and Armadillo (arma.sourceforge.net) package for Linear algebra. We use our own implementation of Lohner's method for continuous transitions and the CSP solving techniques as implemented in the IBEX C++ library (www.ibex-lib.org) for discrete transition. Experiments were conducted on an intel $i 5-3470-3.6 \mathrm{GHz}-16 \mathrm{~GB}$ running Linux.
} 


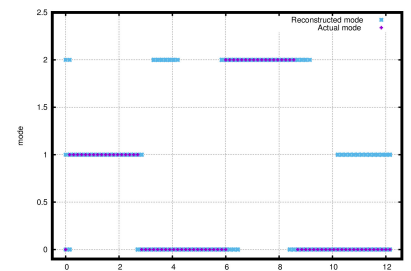

(a) scenario $2: q$

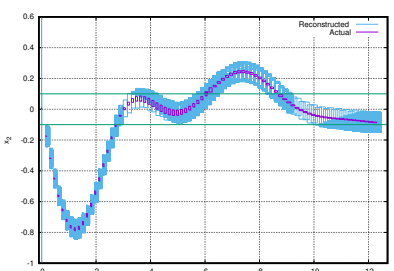

(e) Scenario 2: $x_{2}$

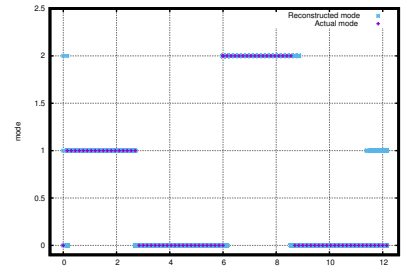

(b) Scenario 1: q

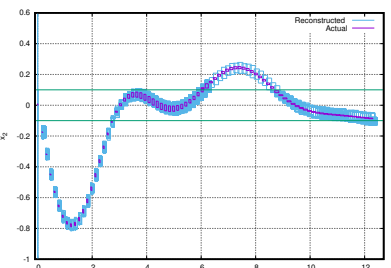

(f) Scenario 1: $x_{2}$

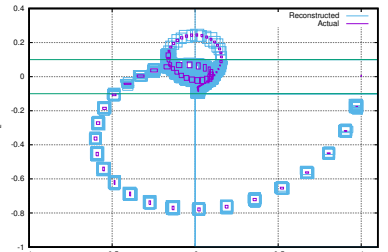

(c) Scenario 1

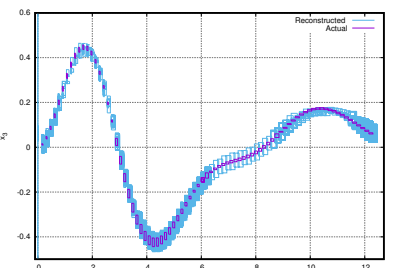

(g) Scenario 1: $x_{3}$

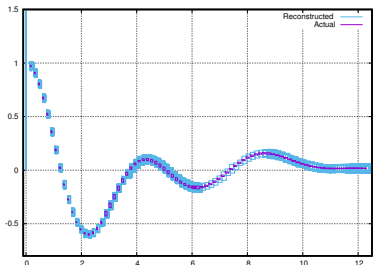

(d) Scenario 1: $x_{1}$

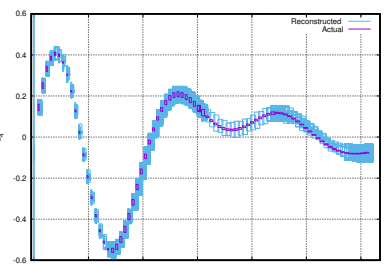

(h) Scenario 1: $x_{4}$

Fig. 2. Scenario $1(\varepsilon=0.025)$ : (b) Active mode estimation, (c) phase portrait $x_{2} \times x_{1}$, time history of estimated variables (d) $x_{1}$, (f) $x_{2},(\mathrm{~g}) x_{3},(\mathrm{~h}) x_{4}$, and. Scenario $2(\varepsilon=0.05)$ : (a) Active mode estimation, time history of estimated variables (e) $x_{2}$.

nonlinear hybrid systems. Then, we have shown how to build a predictor-corrector approach to set-membership state estimation with nonlinear hybrid systems that include guards and jumps. Evaluating our methods with a realistic hybrid system, we were able to successfully analyze and exhibit mode discernibility; then, we were able to reconstruct the switching sequence and the feasible set of continuous state vectors in each discrete location, even if the initial mode were unknown. Future research will analyze applicability of the approaches to fault detection and isolation, and also investigate ways to extend the predictor-corrector approach to event-triggered estimation.

\section{Acknowledgement}

The authors would like to acknowledge the contribution of Moussa Maïga in preparing the numerical evaluation.

\section{References}

T. Alamo, J. Bravo, and E. Camacho. Guaranteed state estimation by zonotopes. Automatica, 41:1035-1043, 2005.

R. Alur, C. Courcoubetis, N. Halbwachs, T. Henzinger, P.-H. Ho, X. Nicollin, A. Olivero, J. Sifakis, and S. Yovine. The algorithmic analysis of hybrid systems. Theoretical Computer Science, 138:3-34, 1995.

M. Babaali and G. J. Pappas. Observability of switched linear systems in continuous time. In LNCS 3414, HSCC, pages 103-117, 2005.

A. Balluchi, L. Benvenuti, Maria D. Di Benedetto, and A. Sangiovanni-Vincentelli. The design of dynamical observers for hybrid systems: Theory and application to an automotive control problem. Automatica, 49: 915-925, 2013.
N. Barhoumi, F. Msahli, M. Djemaï, and K. Busawon. Observer design for some classes of uniformly observable nonlinear hybrid systems. Nonlinear Analysis: Hybrid Systems, 6:917-929, 2012.

E. Benazera and L. Travé-Massuyès. Set-theoretic estimation of hybrid system configurations. IEEE Transactions on Systems, Man, and Cybernetics, Part B, 39(5):1277-1291, 2009.

F. Boulier, D. Lazard, F. Ollivier, and M. Petitot. Computing representations for the radicals of a finitely generated differential ideals. Technical report IT306, LIFL, University of Lille, 1997.

E. De Santis. On location observability notions for switching systems. Systems \& Control Letters, 60(10): $807-814,2011$.

E. De Santis and M. D. Di Benedetto. Observability and diagnosability of finite state systems: A unifying framework. Automatica, 81:115 - 122, 2017.

E. De Santis, M. D. Di Benedetto, and G. Pola. On observability and detectability of continuous-time linear switching systems. In 42nd IEEE International Conference on Decision and Control (IEEE Cat. No.03CH37475), pages 5777-5782 Vol.6, Dec 2003.

E. De Santis, M. D. Di Benedetto, and G. Pola. A structural approach to detectability for a class of hybrid systems. Automatica, 45(5):1202 - 1206, 2009.

L. Denis-Vidal, G. Joly-Blanchard, and C. Noiret. Some effective approaches to check identifiability of uncontrolled nonlinear systems. Mathematics and Computers in Simulation, 57:35-44, 2001.

D. Efimov, T. Raïssi, S. Chebotarev, and A. Zolghadri. Interval state observer for nonlinear time varying systems. Automatica, 49(1):200-205, 2013.

A. Eggers, N. Ramdani, N. S.Nedialkov, and M. Fränzle. Set-membership estimation of hybrid systems via SAT mod ODE. In 16th IFAC SYSID 2012, pages 440-445, 2012. 
M. Fliess, C. Join, and W. Perruquetti. Real-time estimation for switched linear systems. In 2008 47th IEEE Conference on Decision and Control, pages 941-946, Dec 2008.

J. Gouzé, A. Rapaport, and Z. M. Hadj-Sadok. Interval observers for uncertain biological systems. Journal of Ecological Modelling, 133:45-56, 2000.

Y. Guo and B. Huang. Moving horizon estimation for switching nonlinear systems. Automatica, 49:32703281, 2013.

L. Jaulin. Nonlinear bounded-error state estimation of continuous-time systems. Automatica, 38:1079-1082, 2002.

M. Kieffer, L. Jaulin, and E. Walter. Guaranteed recursive non-linear state bounding using interval analysis. International Journal of Adaptative Control and Signal Processing, 16:193-218, 2002.

G. Koch and T. Kloiber. Driving state adaptive control of an active vehicle suspension system. IEEE Transactions on Control Systems Technology, 22(1): 44-57, 2014.

E. Kolchin. Differential algebra and algebraic groups. Academic Press, New York, 1973.

L. Ljung and T. Glad. On global identifiability for arbitrary model parametrizations. Automatica, 30:265276, 1994.

H. Lou and R. Yang. Conditions for distinguishability and observability of switched linear systems. Nonlinear Analysis: Hybrid Systems, 5(3):427 - 445, 2011.

M. Maïga, N. Ramdani, L. Travé-Massuyès, and C. Combastel. A comprehensive method for reachability analysis of uncertain nonlinear hybrid systems. IEEE Transactions on Automatic Control, 61(9):2341- $\overline{2356,}$ 2016.

F. Mazenc and T. N. Dinh. Construction of interval observers for continuous-time systems with discrete measurements. Automatica, 50(10):2555 - 2560, 2014.

F. Mazenc, V. Andrieu, and M. Malisoff. Design of continuousdiscrete observers for time-varying nonlinear systems. Automatica, 57:135 - 144, 2015.

N. Meslem and N. Ramdani. Interval observer design based on nonlinear hybridization and practical stability analysis. International Journal of Adaptive Control and Signal Processing, 25(3):228-248, 2011.

N. Meslem, N. Ramdani, and Y. Candau. Using hybrid automata for set-membership state estimation with uncertain nonlinear continuous-time systems. Journal of Process Control, 20:481-489, 2010.

M. Milanese, J. Norton, H. Piet-Lahanier, and E. W. (Eds.). Bounding Approaches to System Identification. Plenum Press, New York, 1996.

T. Raïssi, N. Ramdani, and Y. Candau. Set membership state and parameter estimation for systems described by nonlinear differential equations. Automatica, 40(10):1771-1777, 2004.

T. Raïssi, D. Efimov, and A. Zolghadri. Interval state estimation for a class of nonlinear systems. Automatic Control, IEEE Transactions on, 57(1):260 $\overline{-265,2012 .}$
F. Schweppe. Recursive state estimation: unknown but bounded errors and system inputs. IEEE Trans. On Automatic Control, 13(1):22-28, 1968.

R. E. H. Thabet, T. Raïssi, C. Combastel, D. Efimov, and A. Zolghadri. An effective method to interval observer design for time-varying systems. Automatica, 50:2677-2684, 2014.

H. Tuy. Handbook of global optimization, D.C. Optimization: Theory, Methods and Algorithms (pp. 149-216). Dordrect: Kluwer Academic Publishers, 1995.

A. Vicino and G. Zappa. Sequential approximation of feasible parameter sets for identification with set membership uncertainty. IEEE Transactions on Automatic Control, 41(6):774-785, 1996.

W. Wang, L. Li, D. Zhou, and K. Liu. Robust state estimation and fault diagnosis for uncertain hybrid nonlinear systems. Nonlinear Analysis: Hybrid Systems, $1(1): 2-15,2007$.

J. Xiong, C. Jauberthie, L. Travé-Massuyès, and F. L. Gall. Fault detection by using interval Kalman filtering and constraint propagation approach. In 52th IEEE Conference on Decision and Control, Firenze (Italy), pages 490-495, 2013. 